\title{
Molecular Characterization of Bumble Bees Species from North East Himalayas
}

\author{
Ritu Ranjan Taye ${ }^{1 *}$, Ataur Rahman ${ }^{2}$, Priyabrata Sen $^{2}$, Shimantini Borkataki $^{2}$ \\ and Manha Bathari ${ }^{3}$ \\ ${ }^{1}$ Regional Agricultural Research Station, Karimganj, Assam, India \\ ${ }^{2}$ College of Agriculture, Assam Agricultural University, Jorhat, Assam, India \\ ${ }^{3}$ Regional Agricultural Research Station, Diphu, Assam, India \\ *Corresponding author
}

\section{A B S T R A C T}

\begin{tabular}{|l|}
\hline K e y w o r d s \\
Bumble bee, \\
Molecular \\
characterization, \\
North east \\
Himalayas, \\
Dendrogram \\
\hline Article Info \\
\hline Accepted: \\
15 April 2020 \\
Available Online: \\
10 May 2020 \\
\hline
\end{tabular}

\section{Keywords}

Bumble bee,

Molecular

characterization

North east

Article Info

Accepted:

Available Online

10 May 2020
Molecular characterization of bumble bees has been carried out in the Department of Entomology, Assam Agricultural University during the period 2015-2018. During the investigation, 5 (Five) species of bumble bees viz. Bombus orientalis Smith, B. buccinatoris Smith, B. tunicatus Smith, B. haemorrhoidalis Smith and B. miniatus Bingham have been recorded. Similarity matrix for Jaccard's Coefficient based on SSR banding of 5 bumble bee species ranged from 0.062 to 0.921 i.e. $6.2 \%$ to $92.1 \%$. The dendrogram readily separated the bumble bee species into two main clusters (A and B). The cluster A includes Bombus orientalis and B. buccinatori while cluster B includes B. tunicatus, B. haemorrhoidalis and B. miniatus.

\section{Introduction}

Bumble bees (genus Bombus) are large, colorful, ubiquitous pollinators found throughout the holarctic, oriental, and neotropical regions of the world, especially in alpine and arctic zones. The genus Bombus, comprises over two hundred and fifty (250) known species of bumblebees present on global basis (Williams et al., 2008). Bingham (1897) included 24 species of Bombus in Fauna of British India that included records from India and the neighboring countries; such as Myanmar, Bhutan, Nepal, Sri Lanka etc. The generic name Bombus, assigned by Latreille in 1802, is derived from the Latin word for a buzzing or humming sound. Bumblebees rank among the most abundant 
and conspicuous of flower visitors in alpine, temperate and arctic environment of the northern hemisphere. They are called primitively eusocial and are associated with the high lands and play a key role in the functioning of agricultural ecosystems as pollinators of crops, orchards and wild flowers.

Thus, they serve as important pollinators, especially in alpine environments and early in the flowering season (Kevan and Baker, 1983; $\mathrm{Yu}$ et al., 2012). Bumble bees (Bombus spp.) can be used in greenhouses for pollination services, because they are very efficient pollinators that can be handled with great ease. Bumble bees exceed other pollinator species in pollination efficiency, due to their behavioural particularity of "buzz pollination" (Buchmann, 1985).

In molecular study, the relationships among organisms or genes are studied by comparing homologues of DNA or protein sequences. Dissimilarities among the sequences indicate genetic divergence as a result of molecular evolution during the course of time. . By comparing homologous molecules from different organisms it is possible to establish their degree of similarity thereby establishing or revealing a hierarchy of relationship a phylogenetic tree. Both the classical morphology based methods and molecular analysis based methods are of importance as the basic bio-molecular framework of all organisms are similar and morphology of an organism is actually the manifestations of its genome, proteome and transcriptome profiles.

\section{Materials and Methods}

\section{Sample collection}

North east Himalaya is located at $25.5736^{\circ} \mathrm{N}$ latitude and $93.2473^{\circ} \mathrm{E}$ longitude in Indian sub-continent. Extensive exploration for bumble bees have been carried out in 5 (five) physiographic zones of north east Himalaya viz. Arunachal Himalaya, Barak valley, Brahmaputra valley, Meghalaya Plateau and South eastern hill tract during 2014-18.

Each physiographic zone had been subdivided into different locations based on Global Positioning System (Table 1). Bee samples were collected based on random sampling methods, covering different forest and agro-ecosystems. The samples were preserved in $75 \%$ ethanol to prevent any kind of deformation.

\section{Molecular analysis}

DNA extraction was done from the thorax of the bee. The homogenous mix was prepared after grinding the thorax with CTAB buffer and $25 \mu \mathrm{l}$ of $10 \mathrm{~g} / \mathrm{ml}$ proteinase $\mathrm{K}$ were added.

The homogenous mix had been centrifuged at $14,000 \mathrm{rpm}$ for 15 minutes at $4^{\circ} \mathrm{C}$ and then $500 \mu 1$ of upper phase transferred to another sterile tube, mixed with equal volume of chloroform: Isoamyl alcohol $\left(24^{\circ} .1\right)$. The mix was stored at $-20^{\circ} \mathrm{C}$ for overnight and had been centrifuged at $14,000 \mathrm{rpm}$ for 15 minutes.

Isolated DNA was quantified by using Nanodrop 200UV- visible spectrophotometer and electrophosed on 0.85 agarose gel. The DNA amplification was achieved by use of synthetic oligonucleotides termed primers that are forward and reverse primer (Table 2).

DNA polymerase was then used to carry out the synthesis of a complementary strand of DNA in the $5^{\prime}$ to $3^{\prime}$ direction of sense and antisense strands using the oligonucleotides primer. PCR reactions require typically three phases: firstly the DNA template has to be denatured $\left(92-94^{\circ} \mathrm{C}\right)$; secondly the primers have to be annealed $\left(40-65^{\circ} \mathrm{C}\right)$ and thirdly 
DNA polymerase extends the annealed primers along the single-stranded template $\left(72^{\circ} \mathrm{C}\right)$. The molecular weight of PCR products, obtained for each marker was designated, based on a ladder of known molecular weight. Data was scored on the basis of presence or absence of the amplified particular DNA fragment products.

\section{Statistical analysis}

An agglomerative method of clustering genotype was employed utilizing the Unweighted Pair Group Method with Arithmetic averages (UPGMA). The relationship between species was presented graphically in the form of dendrogram and matrix.

\section{Results and Discussion}

Molecular characterization of bumble bees had been carried out to study the variations at molecular level as well as to obtain molecular markers that can distinguish the species from one another. In the present study, 20 SSR markers were used of which 14 showed banding patters (Table 3). Similarity matrix for Jaccard's Coefficient based on SSR banding of 5 bumble bee species ranged from 0.062 to 0.921 i.e. $6.2 \%$ to $92.1 \%$. The lowest similarity value was found between Bombus haemorrhoidalis from Maibang and $B$. orientalis from Pasighat (6.2\%) and the highest similarity value was found $B$. orientalis from Basar and Pasighat (92.1\%).

Table.1 Physiographic zones of North East India showing locations

\begin{tabular}{|c|c|c|c|c|}
\hline Physiographic Zone & Location & Latitude & Longitude & Elevation \\
\hline \multirow[t]{5}{*}{ Arunachal Himalaya } & Pasighat & $28.07^{\circ} \mathrm{N}$ & $95.33^{\circ} \mathrm{E}$ & $155 \mathrm{~m}$ \\
\hline & Roing & $28.14^{\circ} \mathrm{N}$ & $95.84^{\circ} \mathrm{E}$ & $390 \mathrm{~m}$ \\
\hline & Basar & $27.98^{\circ} \mathrm{N}$ & $94.66^{\circ} \mathrm{E}$ & $578 \mathrm{~m}$ \\
\hline & Itanagar & $27.1^{\circ} \mathrm{N}$ & $93.62^{\circ} \mathrm{E}$ & $750 \mathrm{~m}$ \\
\hline & Hawai & $27^{\circ} 53 \mathrm{~N}$ & $96^{\circ} 48 \mathrm{E}$ & $1296 \mathrm{~m}$ \\
\hline \multirow[t]{5}{*}{ Barak valley } & Agartala & $23.83^{\circ} \mathrm{N}$ & $91.26^{\circ} \mathrm{E}$ & $12 \mathrm{~m}$ \\
\hline & Karimganj & $24.86^{\circ} \mathrm{N}$ & $92.36^{\circ} \mathrm{E}$ & $13 m$ \\
\hline & Udaipur & $23.53^{\circ} \mathrm{N}$ & $91.48^{\circ} \mathrm{E}$ & $22 \mathrm{~m}$ \\
\hline & Kailashahar & $24.33^{\circ} \mathrm{N}$ & $92.02^{\circ} \mathrm{E}$ & $24 \mathrm{~m}$ \\
\hline & Cachar & $24.78^{\circ} \mathrm{N}$ & $92.86^{\circ} \mathrm{E}$ & $25 \mathrm{~m}$ \\
\hline \multirow[t]{5}{*}{ Brahmaputra Valley } & Jorhat & $26.75^{\circ} \mathrm{N}$ & $94.20^{\circ} \mathrm{E}$ & $93 m$ \\
\hline & North Lakhimpur & $27.24^{\circ} \mathrm{N}$ & $94.11^{\circ} \mathrm{E}$ & $96 \mathrm{~m}$ \\
\hline & Golaghat & $26.59^{\circ} \mathrm{N}$ & $93.75^{\circ} \mathrm{E}$ & $98 \mathrm{~m}$ \\
\hline & Nagaon & $26.57^{\circ} \mathrm{N}$ & $93.00^{\circ} \mathrm{E}$ & $70 \mathrm{~m}$ \\
\hline & Dibrugarh & $27.47^{\circ} \mathrm{N}$ & $94.92^{\circ} \mathrm{E}$ & $110 \mathrm{~m}$ \\
\hline \multirow[t]{5}{*}{ Meghalaya plateau } & Umragnso & $25.51^{\circ} \mathrm{N}$ & $92.73^{\circ} \mathrm{E}$ & $640 \mathrm{~m}$ \\
\hline & Umsning & $25.75^{\circ} \mathrm{N}$ & $91.89^{\circ} \mathrm{E}$ & $782 \mathrm{~m}$ \\
\hline & Umiam & $25.67^{\circ} \mathrm{N}$ & $91.89^{\circ} \mathrm{E}$ & $946 \mathrm{~m}$ \\
\hline & Sohra & $25.28^{\circ} \mathrm{N}$ & $91.73^{\circ} \mathrm{E}$ & $1484 m$ \\
\hline & Sanmer & $25.55 \mathrm{~N}$ & $91.84^{\circ} \mathrm{E}$ & $1726 m$ \\
\hline \multirow{5}{*}{$\begin{array}{l}\text { South Eastern Hill } \\
\text { Tract }\end{array}$} & Maibang & $25.30^{\circ} \mathrm{N}$ & $93.13^{\circ} \mathrm{E}$ & $355 \mathrm{~m}$ \\
\hline & Medziphema & $25.76^{\circ} \mathrm{N}$ & $93.87^{\circ} \mathrm{E}$ & $456 \mathrm{~m}$ \\
\hline & Imphal & $24.81^{\circ} \mathrm{N}$ & $93.90^{\circ} \mathrm{E}$ & $786 \mathrm{~m}$ \\
\hline & Haflong & $25.16^{\circ} \mathrm{N}$ & $93.01^{\circ} \mathrm{E}$ & $966 \mathrm{~m}$ \\
\hline & Kohima & $25.66^{\circ} \mathrm{N}$ & $94.11^{\circ} \mathrm{E}$ & $1445 m$ \\
\hline
\end{tabular}


Table.2 Primer sequences used for PCR amplification in bumble bees

\begin{tabular}{|c|c|c|}
\hline Locus & Position & Primers $\left(5^{\prime}-3^{\prime}\right)$ \\
\hline \multirow[t]{2}{*}{ BT01 } & $\mathrm{F}$ & CCGATCTGTGAGAATGACAGTATCG \\
\hline & $\mathrm{R}$ & CGTGTTTCGATTAGCAAAGCTACG \\
\hline \multirow[t]{2}{*}{ BT02 } & $\mathrm{F}$ & AGGAACCGAGCGATAGAACCAC \\
\hline & $\mathrm{R}$ & GCTTTGCCTTTCCATCTTGCTG \\
\hline \multirow[t]{2}{*}{ BT04 } & $\mathrm{F}$ & GAGAGAGATCGAATGGTGAGAGC \\
\hline & $\mathrm{R}$ & TGAGCACGTTCTTTCGTTCAC \\
\hline \multirow{2}{*}{ BT05 } & $\mathrm{F}$ & TTTCCTATGCCGAACGTCACC \\
\hline & $\mathrm{R}$ & CCCAGATAAAAGACCGCCTCTAGTC \\
\hline \multirow[t]{2}{*}{ BT08 } & $\mathrm{F}$ & AGAACCTCCGTATCCCTTCG \\
\hline & $\mathrm{R}$ & AGCCTACCCAGTGCTGAAAC \\
\hline \multirow[t]{2}{*}{ BT10 } & $\mathrm{F}$ & TCTTGCTATCCACCACCCGC \\
\hline & $\mathrm{R}$ & GGACAGAAGCATAGACGCACCG \\
\hline \multirow{2}{*}{ BT16 } & $\mathrm{F}$ & CAGCCAAAAAATCAGTGGAGTGC \\
\hline & $\mathrm{R}$ & TCTTCCTCTGTTTCTCGTTCACG \\
\hline \multirow{2}{*}{ BT17 } & $\mathrm{F}$ & GCGGATGCACGATATAAAATG \\
\hline & $\mathrm{R}$ & ТСТTТСТСТССТТСТТСССАТС \\
\hline \multirow{2}{*}{ BT20 } & $\mathrm{F}$ & TTCCACAGCGTTTTCTTAAGTC \\
\hline & $\mathrm{R}$ & ATGGACGGCGAGATCGTGAG \\
\hline \multirow{2}{*}{ BT23 } & $\mathrm{F}$ & GCAACAGAAAATCGTCGGTAGTG \\
\hline & $\mathrm{R}$ & GCGGCAATAAAGCAATCGG \\
\hline \multirow[t]{2}{*}{ BT24 } & $\mathrm{F}$ & TCTTTCCGTTTTCCCCCTG \\
\hline & $\mathrm{R}$ & САСССАСТТАСАТАСАТАСАСGСТС \\
\hline \multirow{2}{*}{ BT28 } & $\mathrm{F}$ & TTGCTGACGTTGCTGTGACTGAGG \\
\hline & $\mathrm{R}$ & TCСTCTGTGTGTTCTCTTACTTGGC \\
\hline \multirow{2}{*}{ BL01 } & $\mathrm{F}$ & GCGTCGAGAAACTATCTAGGAGAG \\
\hline & $\mathrm{R}$ & CGAAGATTCCCAAAACTGCG \\
\hline \multirow{2}{*}{ BL02 } & $\mathrm{F}$ & GAACAGTGAGAGCGAGGAACAGAG \\
\hline & $\mathrm{R}$ & TTGCCACGTATATCCGAGCGAACC \\
\hline \multirow[t]{2}{*}{ BL05 } & $\mathrm{F}$ & CGAAAATCAGGGGTGACAAAC \\
\hline & $\mathrm{R}$ & CCTTTCTGTTTATAGTTCGTCCG \\
\hline \multirow[t]{2}{*}{ BL08 } & $\mathrm{F}$ & ATGTTGCAGCACCTTCGTGG \\
\hline & $\mathrm{R}$ & AATTAAAGGCGTGCGCTCGC \\
\hline \multirow[t]{2}{*}{ BL11 } & $\mathrm{F}$ & AAGGGTACGAAATGCGCGAG \\
\hline & $\mathrm{R}$ & TGACGAGTGCGGCCTTTTTC \\
\hline \multirow[t]{2}{*}{ BL13 } & $\mathrm{F}$ & CGAATGTTGGGATTTTCGTG \\
\hline & $\mathrm{R}$ & GCGAGTACGTGTACGTGTTCTATG \\
\hline \multirow{2}{*}{ BTERN01 } & $\mathrm{F}$ & CGTGTTTAGGGTACTGGTGGTC \\
\hline & $\mathrm{R}$ & GGAGCAAGAGGGCTAGACAAAAG \\
\hline \multirow{2}{*}{ BTERN02 } & $\mathrm{F}$ & TTTCCACССТTCACGCATACAC \\
\hline & $\mathrm{R}$ & GATTTTATCCTCCGACCGTTCC \\
\hline
\end{tabular}


Table.3 Similarity matrix of Jaccard's coefficient of dwarf bees from different physiographic zones

\begin{tabular}{|c|c|c|c|c|c|c|c|c|c|c|c|c|c|c|c|c|c|c|c|c|c|c|c|c|c|c|c|c|}
\hline & 1 & 2 & 3 & 4 & 5 & 6 & 7 & 8 & 9 & 10 & 11 & 12 & 13 & 14 & 15 & 16 & 17 & 18 & 19 & 20 & 21 & 22 & 23 & 24 & 25 & 26 & 27 & 28 \\
\hline 1 & 1.000 & & & & & & & & & & & & & & & & & & & & & & & & & & & \\
\hline 2 & 0.921 & 1.000 & & & & & & & & & & & & & & & & & & & & & & & & & & \\
\hline 3 & 0.866 & 0.866 & 1.000 & & & & & & & & & & & & & & & & & & & & & & & & & \\
\hline 4 & 0.429 & 0.429 & 0.577 & 1.000 & & & & & & & & & & & & & & & & & & & & & & & & \\
\hline 5 & 0.577 & 0.577 & 0.708 & 0.866 & 1.000 & & & & & & & & & & & & & & & & & & & & & & & \\
\hline 6 & 0.143 & 0.143 & 0.289 & 0.714 & 0.577 & 1.000 & & & & & & & & & & & & & & & & & & & & & & \\
\hline 7 & 0.289 & 0.289 & 0.167 & 0.577 & 0.458 & 0.866 & 1.000 & & & & & & & & & & & & & & & & & & & & & \\
\hline 8 & 0.577 & 0.577 & 0.458 & 0.289 & 0.458 & 0.289 & 0.417 & 1.000 & & & & & & & & & & & & & & & & & & & & \\
\hline 9 & 0.577 & 0.577 & 0.458 & 0.234 & 0.167 & 0.615 & 0.125 & 0.708 & 1.000 & & & & & & & & & & & & & & & & & & & \\
\hline 10 & 0.577 & 0.577 & 0.458 & 0.282 & 0.167 & 0.615 & 0.125 & 0.708 & 0.781 & 1.000 & & & & & & & & & & & & & & & & & & \\
\hline 11 & 0.289 & 0.289 & 0.458 & 0.289 & 0.458 & 0.602 & 0.167 & 0.125 & 0.417 & 0.417 & 1.000 & & & & & & & & & & & & & & & & & \\
\hline 12 & 0.289 & 0.289 & 0.458 & 0.289 & 0.458 & 0.602 & 0.167 & 0.125 & 0.417 & 0.417 & 0.874 & 1.000 & & & & & & & & & & & & & & & & \\
\hline 13 & 0.174 & 0.174 & 0.101 & 0.174 & 0.101 & 0.174 & 0.251 & 0.251 & 0.251 & 0.251 & 0.251 & 0.251 & 1.000 & & & & & & & & & & & & & & & \\
\hline 14 & 0.167 & 0.522 & 0.091 & 0.090 & 0.091 & 0.316 & 0.411 & 0.291 & 0.391 & 0.791 & 0.391 & 0.234 & 0.826 & 1.000 & & & & & & & & & & & & & & \\
\hline 15 & 0.149 & 0.149 & 0.343 & 0.149 & 0.343 & 0.149 & 0.258 & 0.258 & 0.258 & 0.258 & 0.258 & 0.258 & 0.701 & 0.849 & 1.000 & & & & & & & & & & & & & \\
\hline 16 & 0.289 & 0.289 & 0.167 & 0.289 & 0.167 & 0.289 & 0.417 & 0.125 & 0.125 & 0.125 & 0.125 & 0.125 & 0.603 & 0.730 & 0.559 & 1.000 & & & & & & & & & & & & \\
\hline 17 & 0.289 & 0.289 & 0.167 & 0.289 & 0.167 & 0.289 & 0.417 & 0.125 & 0.125 & 0.125 & 0.125 & 0.125 & 0.603 & 0.730 & 0.559 & 0.678 & 1.000 & & & & & & & & & & & \\
\hline 18 & 0.577 & 0.577 & 0.417 & 0.289 & 0.417 & 0.289 & 0.458 & 0.167 & 0.167 & 0.167 & 0.167 & 0.167 & 0.452 & 0.548 & 0.645 & 0.750 & 0.750 & 1.000 & & & & & & & & & & \\
\hline 19 & 0.149 & 0.149 & 0.430 & 0.149 & 0.430 & 0.447 & 0.559 & 0.258 & 0.258 & 0.258 & 0.643 & 0.493 & 0.701 & 0.849 & 0.689 & 0.861 & 0.861 & 0.645 & 1.000 & & & & & & & & & \\
\hline 20 & 0.149 & 0.149 & 0.430 & 0.149 & 0.043 & 0.447 & 0.559 & 0.258 & 0.258 & 0.258 & 0.143 & 0.438 & 0.701 & 0.849 & 0.689 & 0.861 & 0.861 & 0.645 & 0.764 & 1.000 & & & & & & & & \\
\hline 21 & 0.814 & 0.450 & 0.125 & 0.289 & 0.125 & 0.190 & 0.125 & 0.167 & 0.125 & 0.125 & 0.125 & 0.125 & 0.251 & 0.411 & 0.559 & 0.125 & 0.125 & 0.458 & 0.258 & 0.258 & 1.000 & & & & & & & \\
\hline 22 & 0.289 & 0.289 & 0.167 & 0.190 & 0.167 & 0.289 & 0.417 & 0.417 & 0.417 & 0.417 & 0.125 & 0.125 & 0.603 & 0.730 & 0.861 & 0.708 & 0.708 & 0.750 & 0.861 & 0.861 & 0.417 & 1.000 & & & & & & \\
\hline 23 & 0.410 & 0.520 & 0.091 & 0.316 & 0.091 & 0.097 & 0.391 & 0.491 & 0.291 & 0.191 & 0.491 & 0.291 & 0.440 & 0.650 & 0.849 & 0.411 & 0.411 & 0.548 & 0.519 & 0.519 & 0.730 & 0.730 & 1.000 & & & & & \\
\hline 24 & 0.749 & 0.149 & 0.143 & 0.149 & 0.343 & 0.149 & 0.258 & 0.258 & 0.258 & 0.258 & 0.443 & 0.340 & 0.337 & 0.519 & 0.689 & 0.559 & 0.559 & 0.645 & 0.689 & 0.689 & 0.559 & 0.861 & 0.849 & 1.000 & & & & \\
\hline 25 & 0.130 & 0.312 & 0.125 & 0.289 & 0.125 & 0.560 & 0.125 & 0.125 & 0.417 & 0.417 & 0.125 & 0.125 & 0.251 & 0.411 & 0.559 & 0.417 & 0.417 & 0.458 & 0.559 & 0.559 & 0.708 & 0.708 & 0.730 & 0.861 & 1.000 & & & \\
\hline 26 & 0.316 & 0.316 & 0.228 & 0.510 & 0.228 & 0.316 & 0.411 & 0.411 & 0.411 & 0.411 & 0.411 & 0.411 & 0.440 & 0.650 & 0.849 & 0.411 & 0.411 & 0.548 & 0.519 & 0.519 & 0.411 & 0.730 & 0.650 & 0.519 & 0.411 & 1.000 & & \\
\hline 27 & 0.062 & 0.630 & 0.167 & 0.620 & 0.167 & 0.289 & 0.125 & 0.125 & 0.417 & 0.417 & 0.708 & 0.708 & 0.251 & 0.411 & 0.559 & 0.125 & 0.125 & 0.167 & 0.258 & 0.258 & 0.417 & 0.417 & 0.411 & 0.258 & 0.417 & 0.730 & 1.000 & \\
\hline 28 & 0.149 & 0.149 & 0.344 & 0.149 & 0.344 & 0.447 & 0.258 & 0.258 & 0.258 & 0.258 & 0.559 & 0.559 & 0.337 & 0.519 & 0.689 & 0.258 & 0.258 & 0.344 & 0.378 & 0.378 & 0.258 & 0.559 & 0.519 & 0.378 & 0.258 & 0.849 & 0.861 & 1.000 \\
\hline
\end{tabular}

$(\mathrm{BO}=$ Bombus orientalis; $\mathrm{BB}=B$. buccinatoris; $\mathrm{BT}=B$. tunicatus; $\mathrm{BH}=B$. haemorrhoidalis and $\mathrm{BB}=B$. miniatus $)$

\begin{tabular}{|l|l|l|l|l|l|l|}
\hline 1:Pasighat(B.O) & 5:Hawai(B.O) & 9:Umsning(B.O) & 13:Roing(B.B) & 17:Umiam(B.B) & 21:Umsning(B.T) & 25:Maibong(B.T) \\
\hline 2:Basar(B.O) & 6:Haflong(B.O) & 10:Umiam(B.O) & 14:Umrangso(B.B) & 18:Hawai(B.B) & 22:Basar(B.B) & 26:Haflong(B.H) \\
\hline 3:Roing(B.O) & 7:Maibang(B.O) & 11:Sohra(B.O) & 15:Pasighat(B.B) & 19:Haflong(B.B) & 23:Umiam(B.T) & 27:Maibong(B.H) \\
\hline 4:Itanagar(B.O) & 8:Umragnso(B.O) & 12:Sanmer(B.O) & 16:Sohra (B.B) & 20:Maibang(B.B) & 24:Haflong(B.T) & 28:Haflong(B.M) \\
\hline
\end{tabular}




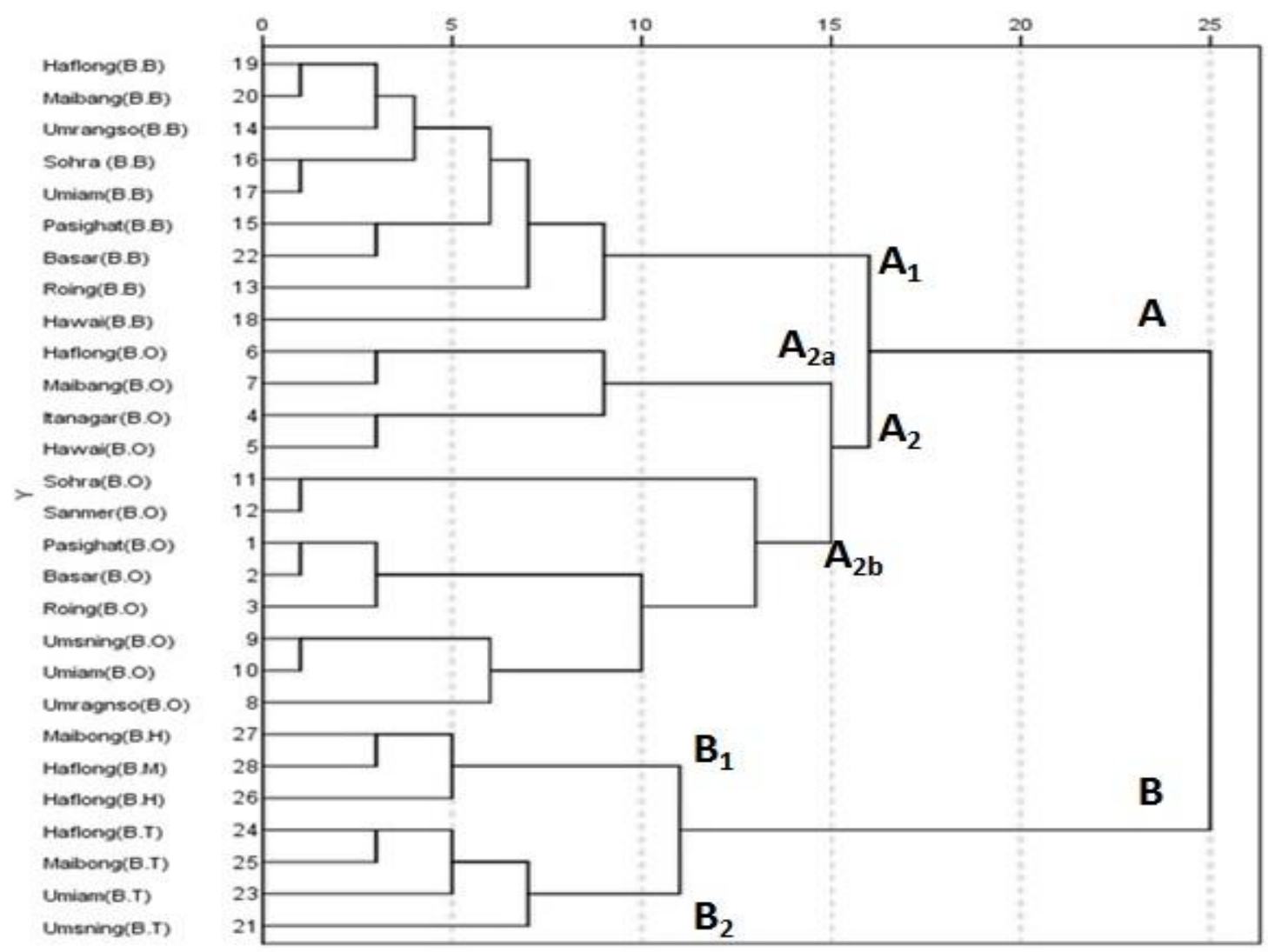

( $\mathrm{BO}=$ Bombus orientalis; $\mathrm{BB}=B$. buccinatoris; $\mathrm{BT}=B$. tunicatus; $\mathrm{BH}=B$. haemorrhoidalis and $\mathrm{BB}=B$. miniatus $)$

Fig.1 Dendrogram showing linkage based on DNA fragment amplified by SSR markers across bumble bee population from different physiographic zones of north east Himalaya

Similarity matrix for Jaccard's Coefficient based on SSR banding of 5 bumble bee species ranged from 0.062 to 0.921 i.e. $6.2 \%$ to $92.1 \%$ (Table 3). The lowest similarity value was found between Bombus haemorrhoidalis from Maibang and $B$. orientalis from Pasighat $(6.2 \%)$ and the highest similarity value was found $B$. orientalis from Basar and Pasighat (92.1 Estoup et. al. (1996) have conducted the studies on Bombus terrestris by using microsatellites and found that there is a high level of intrapopulational polymorphism with tested microsatellite. The dendrogram readily separated the bumble bee species into two main clusters (A and B) (Fig. 1). The cluster A includes Bombus orientalis and $B$. buccinatori while cluster $\mathrm{B}$ includes $B$. tunicatus, $B$. haemorrhoidalis and $B$. miniatus. The Cluster A consists of two sub- clusters $A_{1}$ and $A_{2}$. The sub-cluster $A_{1}$ includes $B$. buccinatori from Haflong, Maibang, Umrangso, Sohra, Umiam, Pasighat, Basar, Roing and Hawai. The subcluster $A_{2}$ is consisting of two sub clusters $A_{2 a}$ and $\mathrm{A}_{2 \mathrm{~b}}$.

The sub cluster $\mathrm{A}_{2 \mathrm{a}}$ includes $B$. orientalis from Haflong, Maibang, Itanagar and Hawai while the sub cluster $A_{2 b}$ again divided into two groups. The first group includes $B$. orientalis from Sohra and Sanmer while second group includes Pasighat, Basar, Roing, Umsning, Umiam and Umrangso. The cluster $\mathrm{B}$ consist of two sub-clusters $\mathrm{B}_{1}$ and $\mathrm{B}_{2}$. The sub cluster $\mathrm{B}_{1} B$. haemorrhoidalis from Haflong and Maibang and B. miniatus from Haflong while sub cluster $\mathrm{B}_{2}$ includes $B$. tunicatus from Haflong, Maibang, Umiam and Umsning. Funk et al., (2006) report the 
details and characteristics of a total 44 novel microsatellite loci for Bombus spp. Most of them were highly polymorphic to $B$. terrestris and a high degree of polymorphism was also found where these primers have been tested in 10 other bumble bee species. The results obtained from this study suggest that there is considerable level of genetic diversity among bumble bee species from north east Himalaya.

\section{References}

Aytekin, A. M., and Çağatay, N. 2003. Systematical Studies on Megabombus (Apidae: Hymenoptera) Species in Central Anatolia. Turk. J. Zool. 27, 195204.

Barkan, N.P., and Aytekin, A.M. 2013. Systematical studies on the species of the subgenus Bombus (Thoracobombus) (Hymenoptera: Apidae, Bombus Latreille) in Turkey. Zootaxa. 3737 (2), 167-183.

Bingham, C.T. 1897. The fauna of British India, including Ceylon and Burma. Hymenoptera. Vol. 1. Wasps and bees. London: Taylor and Francis.

Buchmann, S.L. 1985. Bees use vibration to aid pollen col-lection from nonporicidal flowers. J. Kansas Entomol. Soc. 58, 517-525.

Chauhan, A., Katna, S., and Rana, B.S. 2013. Ethological studies on bumble bees under subtropical conditions in India. J. Med. Sci. Clin. Res. 1(3), 125-129.

Dayal, K., and Rana, B.S. 2007. Morphometrics of queen and workers of the bumble bee, Bombus haemorrhoidalis (Hymenoptera:

Apidae). Ind bee J. 69(1- 4), 103-106.

Estoup, A., Solignac, M., Cornuet, J.M., Goudet, J., and Scholls, A. 1996. Genetic differentiation of continental and island populations of Bombus terrestris (Hymenoptera: Apidae) in Europe. Mol. Ecol. 5, 19-31.

Funk, C.R., Hempel, R.S., and Hempel, P.S. 2006. Microsatellite loci for Bombus spp. Mol. Ecol. Resour. 6(1), 83-86.

Kevan, P.G., and Baker, H.G. 1983. Insects as flower visitors and pollinators. Annu. Rev. Entomol. 28, 407-453.

Medler, J.T. 1962. Morphometric Studies on Bumble Bees. Annals of the Entomol. Soc. Amer. 55(2), 212-218.

Pekkarinen, A. 1979. Morphometric, colour and enzyme variation inn bumblebees (Hymenoptera, Apidae, Bombus) in Fennoscandia and Demark. Acta Zool. Fennica. 158, 60.

Svensson, B.G., and Lundberg, H. 1977. Distribution of bumble bee nests in a subalpine/alpine area in relation to altitude and habitat (Hymenoptera, Apidae). Zoon. 5, 63-72.

Williams, P.H., Cameron, S.A., Hines, H.M., Cederberg, B., and Rasmont, P. 2008. A simplified subgeneric classification of the bumble bees (genus Bombus). Apidologie 39, 1-29.

Yu, W.B., Li, D.Z., and Wang, H.: Highly efficient pollination by bumblebees ensures seed production in Pedicularis lachnoglossa (Orobanchaceae), an early-flowering Himalayan plant. J. Syst. Evol. 50, 218-226.

\section{How to cite this article:}

Ritu Ranjan Taye, Ataur Rahman, Priyabrata Sen, Shimantini Borkataki and Manha Bathari. 2020. Molecular Characterization of Bumble Bees Species from North East Himalayas. Int.J.Curr.Microbiol.App.Sci. 9(05): 2010-2016. doi: https://doi.org/10.20546/ijcmas.2020.905.227 